The Chittagong Univ. J. B. Sci., Vol. 3(1 \&2): pp. 65-76, 2008.

\title{
ESSENTIAL OILS OF LEAVES AND RHIZOMES OF KAEMPFERIA GALANGA LINN.
}

\author{
MD. NAZRUL ISLAM BHUIYAN ${ }^{1}$, JARIPA BEGUM ${ }^{1}$ AND M. N. ANWAR ${ }^{2 *}$ \\ ${ }^{1}$ BCSIR Laboratories, Chittagong; Chittagong-4220, Bangladesh. ${ }^{2}$ Department of Microbiology, \\ University of Chittagong, Chittagong-4331, Bangladesh.
}

\begin{abstract}
Kaempferia galanga Linn. leaf and rhizome oils, obtained by hydrodistillation, were analyzed by gas chromatography mass spectroscopy (GC-MS). One hundred and eight components were identified in the leaf oil. The major components were linoleoyl chloride (21.42\%), caryophyllene oxide (11.75\%), cubenol $(9.66 \%)$ and caryophyllene $(5.60 \%)$. Eighty one components were identified in rhizome oil with the main components being 2-propenoic acid, 3-(4-methoxyphenyl),-ethyl ester $(63.36 \%)$, ethyl cinnamate $(6.31 \%)$, 4-cyclooctene -1-methanol (4.61\%), caryophyllene oxide $(4.37 \%)$ and limonene $(3.22 \%)$. The compositions of both oils varied qualitatively and quantitatively.
\end{abstract}

Key words: Essential oil, GC-MS analysis, linoleoyl chloride, 2-propenoic acid, 3-(4-methoxyphenyl)-ethyl ester.

\section{INTRODUCTION}

Kaempferia galanga Linn. (Synonyms Kaemferia rotunda Blanco, Kaempferia latifolia Hornem.) from the Zingiberaceae family is commonly used as a spice ingredient and medicinal herb in South-East Asia, and is valued traditionally for their skin protectant action.

Its tubers are stimulant, expectorant, diuretic and carminative, coughs and asthma. The leaves are used in lotions and as a poultice for sore eyes, sore throat, swellings, rheumatism and fevers. Rhizome juice is applied by the Chakma of Rangamati district for toothache. In paralysis rhizome paste is applied on legs and arms. Rhizome paste is also applied on forehead in headache. Essential oil of rhizome possesses antifungal properties (Gani 2003). Kaempferia galanga has a peppery camphorous taste (Van Wyk and Ben-Erik 2005). Its alcoholic maceration has also been applied as liniment for rheumatism (Kanjanapothi et al. 2004). According to Burkill (1966), the rhizome is used in cosmetics. Smith and Stuart (1973) say that the rhizome is used in perfumery and also as a means to

\footnotetext{
* Corresponding author. : anwarmn51@ yahoo.com 
preserve clothes from insects. Smith and Stuart also (1973) say that the rhizomes are used as a wash for dandruff or scabs of the head. $K$. galanga rhizome contains about 1.5 to $2 \%$ essential oil, whose main components are ethyl cinnamate (25\%), ethyl p-methoxycinnamate $(30 \%)$ and p-methoxycinnamic acid. $K$. galanga is a good natural source of a biologically active easter compound ethyl pmethoxycinnamate. The antifungal action of ethyl p-methoxycinnamate is reported in the literature. Essential oil from the rhizomes was found active against gram positive and gram-negative microorganisms (Ibrahim et al. 2003, Norajit et al. 2007). Medicinal herbs are an indispensible part of traditional medicine. The rhizome of $K$. galanga finds an important place in indigenous medicine as an expectorant, diuretic and carminative (Achuthan and Jose 1997). It is also found to have anticancer (Xue and Chen 2002), antihypertensive (Othman et al. 2002) and larvicidal and repellent activity (Choochote et al. 1999). It is used for the treatment of various skin disorders, rheumatism, catarrhal infections and diabetes mellitus (Mangaly and Sabu 1991, Vagbhata and Hridayam 1971). Similarly major component, ethyl-trans- $p$-methoxycinnamate is present in both the varieties, its percentage varies (39 and 35\%, respectively) (Indrayan et al. 2007). Philip et al. (1945) reported lower spleen indices in women than men in Southern Madras and the women of the region smeared themselves with galangal (Kaempferia galanga) before bathing. Kiuchi et al. (1987) reported that 3-caren5-one found from $K$. galanga. There are no previous references in literature about these Bangladeshi oils. So this work has been undertaken to determine the chemical composition of leaf and rhizome oils of $K$. galanga. These features allow it to be identified for medicinal use and classified among the other ginger oils available in the international market.

\section{MATERIALS AND METHODS}

\section{PLANT MATERIAL}

The plant materials of Kaempferia galanga was collected from the plants grown in the campus of BCSIR Laboratory, Chittagong, during December 2007. The specimen was identified and one-voucher specimen (Y-984) was deposited in the herbarium of BCSIR Laboratory, Chittagong.

\section{Extraction of essential oil}

Samples of leaf was harvested from healthy, two-year-old plants. Freshly harvested leaves (500 g ) and rhizome (200 g) were grounded in a blender separately. The grounded leaves and rhizome were subjected to hydrodistillation using a modified Clevenger-type glass apparatus for $4 \mathrm{~h}$ for isolation of oils separately. The oil samples were stored at $0^{\circ} \mathrm{C}$ in air-tight containers after drying 
ESSENTIAL OILS OF $K$. GALANGA LINN.

them over anhydrous sodium sulfate and filtered before Undertaking the GC-MS analysis.

\section{GC-MS analysis}

The essential oil from leaves and rhizomes of Kaempferia galanga were analyzed by GC-MS electron impact ionization (EI) method on GC-17A gas chromatograph (Shimadzu) coupled to a GC-MS QP 5050A Mass Spectrometer (Shimadzu); fused silica capillary column $(30 \mathrm{~m} \mathrm{x} 0.25 \mathrm{~mm} ; 0.25 \mu \mathrm{m}$ film thickness), coated with DB-5 (J\&W); column temperature $100^{\circ} \mathrm{C}(2 \mathrm{~min})$ to $250^{\circ} \mathrm{C}$ at the rate of $3^{\circ} \mathrm{C} / \mathrm{min}$; carrier gas, helium at constant pressure of $90 \mathrm{Kpa}$. Acquisition parameters full scan; scan range 40-350 amu.

\section{Identification of the compounds}

Compound identification was done by comparing the NIST library data of the peaks with those reported in literature, mass spectra of the peaks with literature data. Percentage composition was computed from GC peak areas on DB-5 column without applying correction factors.

\section{RESULTS AND DISCUSSION}

Essential oils from the leaves and rhizomes of $K$. galanga from Bangladesh were analyzed by GC-MS. The oil yields were $0.55 \%$ and $1.05 \%$, respectively. The composition of the leaf and rhizome oil of $K$. galanga is shown in table 1 and 2. According to GC-MS analysis under the conditions described above, linoleoyl chloride was detected as the main component $(21.42 \%)$ of the leaf. The remaining constituents were caryophyllene oxide $(11.75 \%)$, cubenol (9.66\%), caryophyllene $(5.60 \%)$, 2-propenoic acid, 3-(4-methoxyphenyl)-, ethyl ester $(5.56 \%)$, isoledene $(4.91 \%)$, ethyl cinnamate $(3.66 \%)$, borneol $(2.86 \%), \delta$ cadinene $(2.23 \%), \delta$-selinene $(2.03 \%)$, Ylangene $(1.98 \%), \alpha$-bulnesene $(1.38 \%)$, seychellene $(1.19 \%)$, $\alpha$-caryophyllene $(1.11 \%)$, pentadecane $(1.10 \%)$ and 6-hexadecen-4-yne, (E)- (1.09\%). On the other hand, rhizome oil contained 2-propenoic acid, 3-(4-methoxyphenyl),-ethyl ester (63.36\%), ethyl cinnamate $(6.31 \%)$, 4-cyclooctene -1-methanol (4.61\%), caryophyllene oxide $(4.37 \%)$, limonene (3.22\%), borneol $(2.46 \%)$, cubenol $(1.67 \%)$ and nerolidyl acetate $(1.05 \%)$. Results showed that the oils were complex mixture of numerous compounds, many of which were present in trace amounts. It is worth mentioning here that there is great variation in the chemical composition of leaves and rhizomes oils. Linoleoyl chloride and 2-propenoic acid, 3-(4-methoxyphenyl),ethyl ester are the main common compounds in leaves and rhizomes' oils. 
On the other hand, comparison of $K$ galanga oils that reported herein with those reported from different places in the world showed that $K$ galanga oil of Bangladesh is especially different from others. Besides, it is very interesting to note that $K$ galanga essential oil constituents that reported from the leaf oil of different places showed different results in the content of some of the major and minor compounds. Linoleoyl chloride and 2- propenoic acid, 3-(4methoxyphenyl), -ethyl ester, were reported herein as major constituents ( $K$ galanga oils) but from other parts of the world these compounds of the oil were reported either absent or present in trace amount. This confirms the variations which is not due to geographic divergence and ecological conditions but is due to different chemotype as reported from Bangladesh. On the basis of the above fact it may be concluded that $K$ galanga of Bangladesh, may be utilized as a source for the isolation of natural Linoleoyl chloride and 2- propenoic acid, 3-(4methoxyphenyl), -ethyl ester. The high concentration of Linoleoyl chloride and 2propenoic acid, 3-(4-methoxyphenyl), -ethyl ester in leaf and rhizome oil makes it potentially useful in the medicines because they exhibit antinociceptive, antiinflammatory (Sulaiman et al. 2008), carminative (Achuthan and Jose 1997), anticancer (Xue and Chen 2002), antihypersensitive (Othman et al. 2002), larvicidal and repellent activity (Choochote et al. 1999). Besides, they also have anti-microbial properties (Ibrahim et al. 2003, Norajit et al. 2007). It is worth noting that the oil of $K$ galanga have been reported to be used in folk medicine in the treatment of skin disorders, rheumastism, asthma, diabetes and catarrhal.

TABLE 1. PERCENT COMPOSITION OF ESSENTIAL OIL OF LEAVES OF KAEMPFERIA GALANGA.

\begin{tabular}{|c|l|c|}
\hline $\begin{array}{l}\text { Peak } \\
\text { No }\end{array}$ & Name of constituents & Percentage \\
\hline 1. & $\alpha$-caryophyllene & 1.11 \\
\hline 2. & Camphor & 0.13 \\
\hline 3. & m-cymene & 0.17 \\
\hline 4. & $\alpha$-pinene. & 0.09 \\
\hline 5. & Camphene & 0.03 \\
\hline 6. & $\beta$-phellandrene & 0.04 \\
\hline 7. & $\beta$-pinene & 0.06 \\
\hline 8. & $\beta$-myrcene & 0.10 \\
\hline 9. & Cyclooctanol & 0.04 \\
\hline 10. & $\alpha$-phellandrene & 0.12 \\
\hline 11. & 3-carene & 0.09 \\
\hline 12. & Limonene & 0.58 \\
\hline
\end{tabular}


ESSENTIAL OILS OF $K$. GALANGA LINN.

\begin{tabular}{|c|c|c|}
\hline 13. & Eucalyptol & 0.30 \\
\hline 14. & Z-ocimene & 0.02 \\
\hline 15. & $\gamma$-terpinene & 0.06 \\
\hline 16. & 1-undecanol & 0.20 \\
\hline 17. & 2-carene & 0.09 \\
\hline 18. & Fenchone & 0.09 \\
\hline 19. & Linalool & 0.20 \\
\hline 20. & Nonanal & 0.05 \\
\hline 21. & $\alpha$-campholenal & 0.04 \\
\hline 22. & trans-pinocarveol & 0.17 \\
\hline 23. & (+)-isomenthone & 0.09 \\
\hline 24. & Umbellulone & 0.18 \\
\hline 25. & Borneol & 2.86 \\
\hline 26. & Menthyl acetate & 0.12 \\
\hline 27. & 4-terpineol & 0.20 \\
\hline 28. & Tridecanedial & 0.12 \\
\hline 29. & Myrtenal & 0.78 \\
\hline 30. & 1-isobutyl-1-methylsiletane & 0.06 \\
\hline 31. & Decanal & 0.25 \\
\hline 32. & Methyl cyclobutyl(methyl)phosphinate & 0.09 \\
\hline 33. & Isothujol & 0.09 \\
\hline 34. & Z,E-2,13-octadecadien-1-ol & 0.21 \\
\hline 35. & Bornyl formate & 0.09 \\
\hline 36. & Isoamyl caproate & 0.13 \\
\hline 37. & Thujone & 0.08 \\
\hline 38. & 2-dodecenal & 0.07 \\
\hline 39. & 2,6-octadienal, 3,7-dimethyl- & 0.18 \\
\hline 40. & Bornyl acetate & 0.33 \\
\hline 41. & Neomenthol & 0.11 \\
\hline 42. & Carveol (fr.1) & 0.04 \\
\hline 43. & Carveol acetate & 0.15 \\
\hline 44. & Terpinyl acetate & 0.15 \\
\hline 45. & $\alpha$-cubebene & 0.36 \\
\hline 46. & 2-undecenal & 0.06 \\
\hline 47. & Ylangene & 1.98 \\
\hline 48. & $\alpha$-bourbonene & 0.23 \\
\hline 49. & $\beta$-elemene, & 0.45 \\
\hline
\end{tabular}


BHUIYAN ET AL,

\begin{tabular}{|c|c|c|}
\hline 50. & $\delta$-selinene & 2.03 \\
\hline 51. & Caryophyllene & 5.60 \\
\hline 52. & Guaiene & 0.29 \\
\hline 53. & $\begin{array}{l}\text { 1,6,10-dodecatriene, 7,11-dimethyl-3- } \\
\text { methylene-, (E) }\end{array}$ & 0.67 \\
\hline 54. & Seychellene & 1.19 \\
\hline 55. & Ethyl cinnamate & 3.66 \\
\hline 56. & 9,12,15-octadecatrien-1-ol, (Z,Z,Z)- & 0.54 \\
\hline 57. & Cubenol & 9.66 \\
\hline 58. & $\alpha$-muurolene & 0.30 \\
\hline 59. & Germacrene D & 0.78 \\
\hline 60. & Thujopsene-I3 & 0.62 \\
\hline 61. & Valencene & 0.27 \\
\hline 62. & Pentadecane & 1.10 \\
\hline 63. & $\gamma$-selinene & 0.24 \\
\hline 64. & $\begin{array}{l}\text { Thiepino[3,2-e]isobenzofuran-1,3-dione, 3a,10b- } \\
\text { dihydro-3a,10b-dimethy }\end{array}$ & 0.21 \\
\hline 65. & $\alpha$-selinene & 0.21 \\
\hline 66. & Isoledene & 4.91 \\
\hline 67. & $\delta$-cadinene & 2.23 \\
\hline 68. & $\begin{array}{l}\text { Ethanone, 1-(1,3a,4,5,6,7-hexahydro-4-hydroxy- } \\
\text { 3,8-dimethyl-5-azulenyl)- }\end{array}$ & 0.71 \\
\hline 69. & $\alpha$-bulnesene & 1.38 \\
\hline 70. & Guaiene & 0.31 \\
\hline 71. & $\alpha$-calacorene & 0.20 \\
\hline 72. & 8-cadinol, & 0.40 \\
\hline 73. & $\gamma$-elemene & 0.30 \\
\hline 74. & Dihomo-.gamma.-linolenic acid & 0.54 \\
\hline 75. & Caryophyllene oxide & 11.75 \\
\hline 76. & (-)-globulol & 0.39 \\
\hline 77. & $\begin{array}{l}\text { Cyclohexanepropanal, 2,2-dimethyl-6- } \\
\text { methylene- }\end{array}$ & 0.27 \\
\hline 78. & $\begin{array}{l}\text { 12-oxabicyclo[9.1.0]dodeca-3,7-diene, 1,5,5,8- } \\
\text { tetramethyl-, [1R-(1R@,3E,7E,11R @)]- }\end{array}$ & 0.74 \\
\hline 79. & Alloaromadendrene oxide-(1) & 0.98 \\
\hline 80. & $\beta$-humulene & 0.68 \\
\hline 81. & $2,3,3 \mathrm{a}, 4,5,6,7,7 \mathrm{a}-\mathrm{octah} y d r o-1 \mathrm{H}-$ & 0.57 \\
\hline
\end{tabular}


ESSENTIAL OILS OF $K$. GALANGA LINN.

\begin{tabular}{|r|l|c|}
\hline & cyclopenta[a]pentalen-7-ol & \\
\hline 82. & tau.-cadinol & 0.57 \\
\hline 83. & $\begin{array}{l}\text { 2-propenoic acid, 3-(4-methoxyphenyl)-, ethyl } \\
\text { ester }\end{array}$ & 5.56 \\
\hline 84. & Nitrofurate & 0.37 \\
\hline 85. & Linoleoyl chloride & 21.42 \\
\hline 86. & Cycloisolongifolene, 8,9-dehydro- & 0.19 \\
\hline 87. & Hydrocotarnine & 0.19 \\
\hline 88. & Acetic acid N'-(3,4-dichloro-phenyl)-hydrazide & 0.26 \\
\hline 89. & Oleyl alcohol & 0.30 \\
\hline 90. & 1-nonadecene & 0.09 \\
\hline 91. & Megastigma-4,6(Z),8(Z)-triene & 0.26 \\
\hline 92. & 6-hexadecen-4-yne, (E)- & 1.09 \\
\hline 93. & Khusilol & 0.05 \\
\hline 94. & Octadecanoic acid, ethyl ester & 0.07 \\
\hline 95. & 2,4a,8,8- & 0.36 \\
\hline & tetramethyldecahydrocyclopropa[d]naphthalene & \\
\hline 96. & 2-pentadecanone, 6,10,14-trimethyl- & 0.43 \\
\hline 97. & Methyl (Z)-5,11,14,17-eicosatetraenoate & 0.06 \\
\hline 98. & 14-methyl-8-hexadecyn-1-ol & 0.07 \\
\hline 99. & Farnesol & 0.04 \\
\hline 100. & Methyl palmitate & 0.05 \\
\hline 101. & Biformene & 0.13 \\
\hline 102. & Ethyl palmitate & 0.17 \\
\hline 103. & 7-hexadecenal, (Z)- & 0.04 \\
\hline 104. & Phytol & 0.16 \\
\hline 105. & cis-vitamin A Aldehyde & 0.12 \\
\hline 106. & Linoleic acid ethyl ester & 0.10 \\
\hline 107. & 3-tetradecanynoic acid & 0.19 \\
\hline 108. & $\beta$-iraldeine & 0.10 \\
\hline & & \\
\hline
\end{tabular}


BHUIYAN ET AL,

TABLE 2.COMPOSITION OF ESSENTIAL OIL OF RHIZOME OF KAEMPFERIA GALANGA.

\begin{tabular}{|r|l|l|}
\hline $\begin{array}{r}\text { Peak. } \\
\text { No. }\end{array}$ & Name of constituents & $\%$ \\
\hline 1. & $\wp$-muurolene & 0.11 \\
\hline 2. & $\wp$-terpinene & 0.03 \\
\hline 3. & 12-oxabicyclo (9.1.0)dodeca, 3,7-diene, 1,5,5,8-tetramethyl & 0.35 \\
\hline 4. & 13-tetradece -11yh -1-ol & 0.29 \\
\hline 5. & 1-undecene & 0.03 \\
\hline 6. & 2-cyclohexen-1-one, 2-Methyl-5-(1-methylethenyl) & 0.21 \\
\hline 7. & 2-propenoic acid, 3-(4-methoxyphenyl),-ethyl ester & 63.36 \\
\hline 8. & 3-octen-1-ol,(2) & 0.02 \\
\hline 9. & $4,4,8$-trimethyl tricyclo [6.3.1.0.(1.5) dodecane-2,7,-diol & 0.17 \\
\hline 10. & 4-cyclooctene -1-methanol & 4.61 \\
\hline 11 & 4-terpineol & 0.19 \\
\hline 12. & 5-nonanol, 5-methyl & 0.11 \\
\hline 13. & 6-octenal, 3,7-dimethyl & 0.04 \\
\hline 14. & Allyl-3-methoxybenzoate & 0.04 \\
\hline 15. & Aromadendrene oxide & 0.06 \\
\hline 16. & $\beta$-cedren-9-L-ol & 0.13 \\
\hline 17. & $\beta$-elemene & 0.07 \\
\hline 18. & Benzone acid , 4-methoxy & 0.04 \\
\hline 19. & $\beta$-linalool & 0.46 \\
\hline 20. & Borneol & 0.04 \\
\hline 21. & Bornyl acetate & 0.03 \\
\hline 22. & Bornyl formate & \\
\hline
\end{tabular}


ESSENTIAL OILS OF $K$. GALANGA LINN.

\begin{tabular}{|r|l|l|}
\hline 23. & $\beta$-pinene & 0.13 \\
\hline 24. & Cadinene & 0.12 \\
\hline 25. & Calamene & 0.19 \\
\hline 26. & Camphene & 0.24 \\
\hline 27. & Carotol & 0.18 \\
\hline 28. & Carvacrol & 0.08 \\
\hline 29. & Carveol & 0.12 \\
\hline 30. & Carvyl acetate & 0.03 \\
\hline 31. & Caryophyllene oxide & 4.37 \\
\hline 32. & Cholestan-3-ol, 2-methylelene (3B,5a) & 0.49 \\
\hline 33. & cis-2-pinanol & 0.07 \\
\hline 34. & Cis-L-copaene -8-ol & 0.11 \\
\hline 35. & cis-sabinenehydrate & 0.03 \\
\hline 36. & Cubenol & 1.67 \\
\hline 37. & Cycloisolongifolene, 9,10-Dehydro & 0.08 \\
\hline 38. & Cyclopentanol, 1-(methylenecyclopropyl) & 0.03 \\
\hline 39. & Dihydrocarveol & 0.14 \\
\hline 40. & E-ocimene & 0.02 \\
\hline 41. & Ethyl cinnamate & 6.31 \\
\hline 42. & Eucalyptol & 0.57 \\
\hline 43. & Eucarvone & 0.03 \\
\hline 44. & Geranyl methyl ether & 0.05 \\
\hline 44. & Guaiacol & 0.43 \\
\hline 46. & Hydrindane & \\
\hline 47. & Isolongifolene, 7,10-dehydro & 0.37 \\
\hline & & \\
\hline
\end{tabular}


BHUIYAN ET AL,.

\begin{tabular}{|c|c|c|}
\hline 48. & Isopulegol & 0.47 \\
\hline 49. & Isopulegol acetate & 0.13 \\
\hline 50. & Jasmone & 0.33 \\
\hline 51. & $\alpha$-bisabolol & 0.07 \\
\hline 52. & $\alpha$-calacorene & 0.20 \\
\hline 53. & Ledol & 0.37 \\
\hline 54. & Limonene & 3.22 \\
\hline 55. & Linalyl iso-valerate & 0.03 \\
\hline 56. & Longipinocarvone & 0.26 \\
\hline 57. & $\alpha$-phellandrene & 0.06 \\
\hline 58. & $\alpha$-pinene & 0.15 \\
\hline 59. & $\alpha$-pinocarveol & 0.05 \\
\hline 60. & $\alpha$-selinene & 0.29 \\
\hline 61. & m-cymene, 5 -tert butyl & 0.11 \\
\hline 62. & Megastigmatrienone & 0.14 \\
\hline 63. & Methyl chavicol & 0.05 \\
\hline 64. & Methyl cinnamate & 0.06 \\
\hline 65. & m-methaxyomandelic acid & 0.88 \\
\hline 66. & Murolan-3,9(11) - diene-10-peroxy & 0.20 \\
\hline 67. & Nerolidyl acetate & 1.05 \\
\hline 68. & O-cymene & 0.15 \\
\hline 69. & $\gamma$-anisaldehyde & 0.50 \\
\hline 70. & $\gamma$-cymen-8-ol & 0.08 \\
\hline 71. & Pentadecane & 0.04 \\
\hline 72. & Phytol & 0.18 \\
\hline
\end{tabular}


ESSENTIAL OILS OF $K$. GALANGA LINN.

\begin{tabular}{|r|l|l|}
\hline 73. & $\gamma$-methoxyhydrocinnamic acid & 0.23 \\
\hline 74. & Tetracyclo[ 6,3,2,0(2.5),0(1,8)] -trdecan-9-ol, 4,4,-dimethyl & 0.73 \\
\hline 75. & Thujone & 0.03 \\
\hline 76. & Thymol & 0.05 \\
\hline 77. & Thymoquinone & 0.07 \\
\hline 78. & Ylangene & 0.09 \\
\hline 79. & Z-ocimene & 0.03 \\
\hline 80. & $\beta$-elemene & 0.07 \\
\hline 81. & $\gamma$-muurolene & 0.31 \\
\hline
\end{tabular}

\section{REFERENCES}

ACHUTHAN, C.R. AND JOSE, P. 1997. Hypolipidemic effect of Alpinia galanga (Rasna) and Kaempferia galanga (Kachoori). Ind. .J Clin. Biochem. 12(1): 55-58.

BURKILL, I.H .1966. A Dictionary of the Economic Products of the Malay Peninsula (Vol. 2). Published on behalf of the Government of Malaysia and Singapore by the Ministry of Agriculture and Cooperatives. Kuala Lumpur, Malaysia. 1296-1297 pp.

CHOOCHOTE, W., KANJANAPOTHI, D., PANTHANGA, A., TAESOTIKUL, T., JITPAKDI, A., CHAITHONG, U. AND PITASAWAT, B. 1999. Larvicidal 'and repellent effects of Kaempferia galanga. Southeast Asian J. Trap. Med. Public Health 30(3): 470-476.

GHANI, A. 2003. Medicinal Plants of Bangladesh with chemical constituents and uses. 2nd edition, Asiatic Society of Bangladesh, 5 old Secretariate road, Nimtali, Dhaka, Bangladesh. 270-271 pp.

IBRAHIM, B. J., MOHD, S. M.V., CHEN, B. C., IAU, L. C. AND NG, L.S. 2003. Antifungal activity of the essential oils of nine Zingiberaceae species. Pharmaceutical biology 41(5): 392-397.

INDRAYAN, A. K., ALICE KURIAN, TYAGI, P. K., AJAT SHATRU AND ANUJ K RATHI .2007.Comparative chemical study of two varieties of attractive medicinal plant Kaempferia galanga Linn. Natural Product Radiance 6: 327-333. 
KANJANAPOTHI, D., PANTHONGAA, N., LERTPRASERTSUKEB, T., TAESOTIKULA, C., RUJJANAWATED, D., KAEWPINITD, R. , SUDTHAYAKORND, W., CHOOCHOTEC, U., CHAITHONGC, A. , JITPAKDIC AND PITASAWAT, B. 2004. "Toxicity of crude rhizome extract of Kaempferia galanga L. (Proh Hom)", J Ethnopharmacology 90 (2-3): 359-365.

KIUCHI, F., NAKAMURA, N. AND TSUDA, Y .1987. 3-caren-5-one from Kaempferia galanga. Phytochem. 26(12): 335-351.

MANGALY, J.K. AND SABU, M. 1991. Ethanobotany of zingiberaceae. Zingiberaceae workshop. Prince of Songkla University, Hat Yai, Thailand. 15-18 Oct; p. 24.

NORAJIT, K., LAOHAKUNJIT, N.AND KERDCHOECHUEN, O. 2007. Antibacterial Effect of Five Zingiberaceae Essential Oils. Molecules 12: 2047-2060.

OTHMAN, R., IBRAHIM, H., MOHD, M.A., AWANG, K., GILANI, A.V. AND MUSTHAFA, M.R. 2002. Vaso relaxant effect of Kaempferia galanga on smooth muscles of rat aorta. Planta Med. 68(7): 655-657.

PHILIP, M.I., RAMAKRISHNA, V. AND VENKAT RAO, V. 1945. Turmeric and vegetable oils as repellents against Anopheline mosquitoes. Indian Med. Gaz. 80: 343-344.

SMITH, F. AND STUART, G. 1973. Chinese Medicinal Herbs. In: The Herbal Pen Ts'ao (Li Shih-Chen ed.), Georgetown Press,San Francisco. 392 p.

SULAIMAN, M.R., ZAKARIA, Z.A., DAUD, I.A., NG, F.N., NG, Y.C. AND HIDAYAT, M.T. 2008. Antinociceptive and anti-inflammatory activities of the aqueous extract of Kaempferia galanga leaves in animal models. Nat. Med. (Tokyo) 62(2): 221-227.

VAGBHATA, A. AND HRIDAYAM, C.S. 1971. Saranga Sundara Commentary, 3rd Chapter. In: Chaukhambha Orientalia (Arun Datta and Hemadri ed.). Varanasi, Delhi. 167-681 pp.

VAN, W.Y.K.AND BEN-ERIK. 2005. Food Plants of the World, Portland, Oregon: Timber Press, Inc, ISBN 0-88192-743-0

XUE, Y. AND CHEN, H. 2002. Study on the anti-carcinogenic effect of three compounds of Kaempferia galanga L. Xei Shen Yan Jiu 31(4): 247$248,251$. 